\title{
Multicast-Supported Fast Handover for Proxy Mobile IPv6
}

\author{
${ }^{1}$ V. Berlin Hency and ${ }^{2}$ D. Sridharan \\ ${ }^{1}$ Department of Information Technology, MIT Campus, Anna University, Chennai, India \\ ${ }^{2}$ Deparment of Electronics and Communication Engineering, CEG Campus, Anna University, Chennai, India
}

Received 2013-03-01, Revised 2013-04-18; Accepted 2013-04-27

\begin{abstract}
Proxy Mobile IPv6 is a network-based mobility management protocol which is attractive as it does not require the participation of mobile nodes in mobility-related signaling. Unlike the host based approaches like Mobile IPv6, in PMIPv6 the Mobility Access Gateway (MAG) is responsible for tracking the movements of Mobile Node (MN) and to initiate the handover process. In this study, a novel method of multicasting the data packets to both the previous and the new MAGs by Local Mobility Anchor (LMA) during the handover process is proposed. The experimental results show that the handover delay is greatly reduced when compared to the existing approaches and the packet loss during the handover process is reduced.
\end{abstract}

Keywords: Network-Based Mobility Management, Proxy Mobile IPv6 (PMIPv6), Multicast, OMNET++

\section{INTRODUCTION}

In today's fast moving world, the number of mobile subscribers has increased exponentially. Different wireless technologies like IEEE $802.11 \mathrm{a} / \mathrm{b} / \mathrm{g}$ Wireless-Fidelity (WiFi), 802.16 World Interoperability for Microwave Access (WiMAX) and General Packet Radio Service provides services to ensure seamless roaming facility, with low handover delay. IETF has standardized many Mobile internet protocols which address the challenging issue of reducing the handover delay and packet loss. There are two categories of these protocols: host-based protocols and network-based protocols. In host-based protocols, the mobile nodes are involved in mobility-related signaling. Hence these protocols require stack modifications in the mobile nodes. But in network-based protocol, since the mobile nodes are not involved in mobility-related signaling, there is no need of stack modifications in mobile nodes. The serving network itself locates the mobile subscriber's point of attachment and maintains its connection as it changes its point of attachment.

In MIPv6, when a MN moves away from its home network, a Care-of-Address (CoA) is assigned to it by the Foreign Agent (FA) in the foreign Network. MN registers its new CoA with its home agent, which binds the MN's IP address and its new CoA. Figure 1 shows the packet delivery in Mobile IP. (1) When a Correspondent Node $(\mathrm{CN})$, sends a packet to a $\mathrm{MN}$, the packet is intercepted by the Home Agent (HA) (2) HA encapsulates the packet and sends it to the FA (3) FA decapsulates the packet and delivers it to the MN. (4) $\mathrm{MN}$ sends the packet directly to the $\mathrm{CN}$.

MIPv6 suffers from a serious drawback of Triangle routing problem: Even if the CN's location is nearer to $\mathrm{MN}$, the packets from $\mathrm{CN}$ should travel through HA. To overcome this problem, Mobile IP route optimization (Perkins and Wang, 1999), provides a mechanism in which the $\mathrm{CN}$ maintains a binding cache entry. When the $\mathrm{CN}$ sends a packet to the MN which is away from the home network and the HA intercepts it, HA also sends a binding update message to the $\mathrm{CN}$ which contains the MN's current $\mathrm{CoA}$. On receiving this, $\mathrm{CN}$ updates its binding cache entry and from then on sending the packet directly to MN's current CoA. Packets that are sent during the handoff process are lost as the $\mathrm{CN}$ delivers the packet to the outdated entry.

In Hierarchical Mobile IPv6 (HMIPv6), the FAs are arranged in a hierarchical manner, with one FA at the root and others in the lower level hierarchy to handle local movements of the MN. Figure 2 shows the network of hierarchical FAs. When the $\mathrm{MN}$ is away from the home network, it registers the vector of CoAs, IP address of the current FA as well as with all its ancestors, with the HA.

Corresponding Author: V. Berlin Hency, Department of Information Technology, MIT Campus, Anna University, Chennai, India 


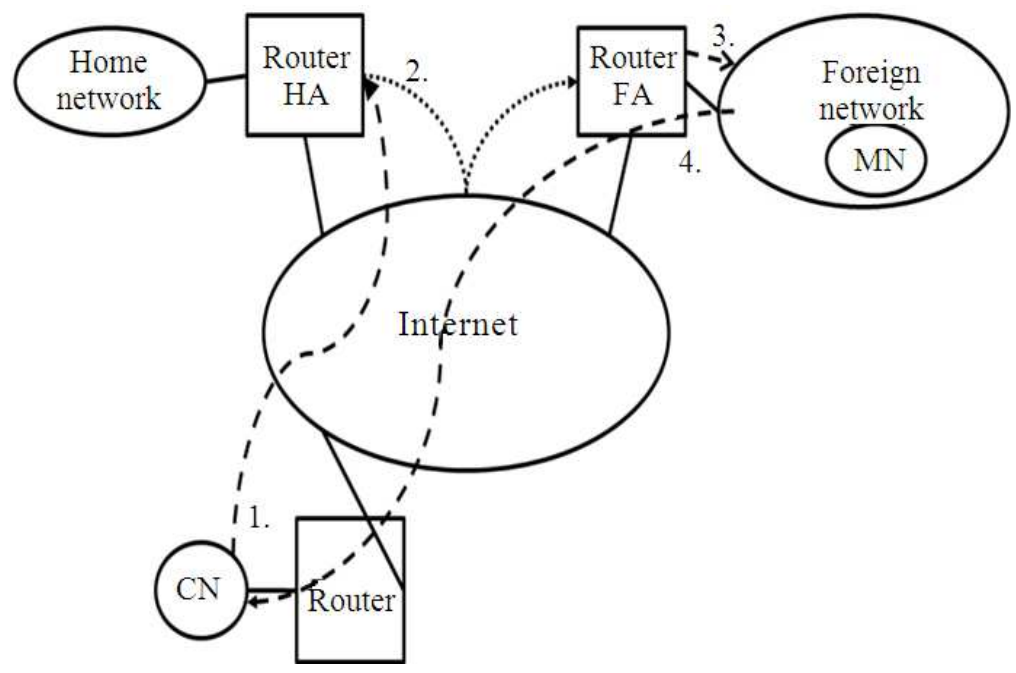

Fig. 1. IP packet delivery in mobile IPv6

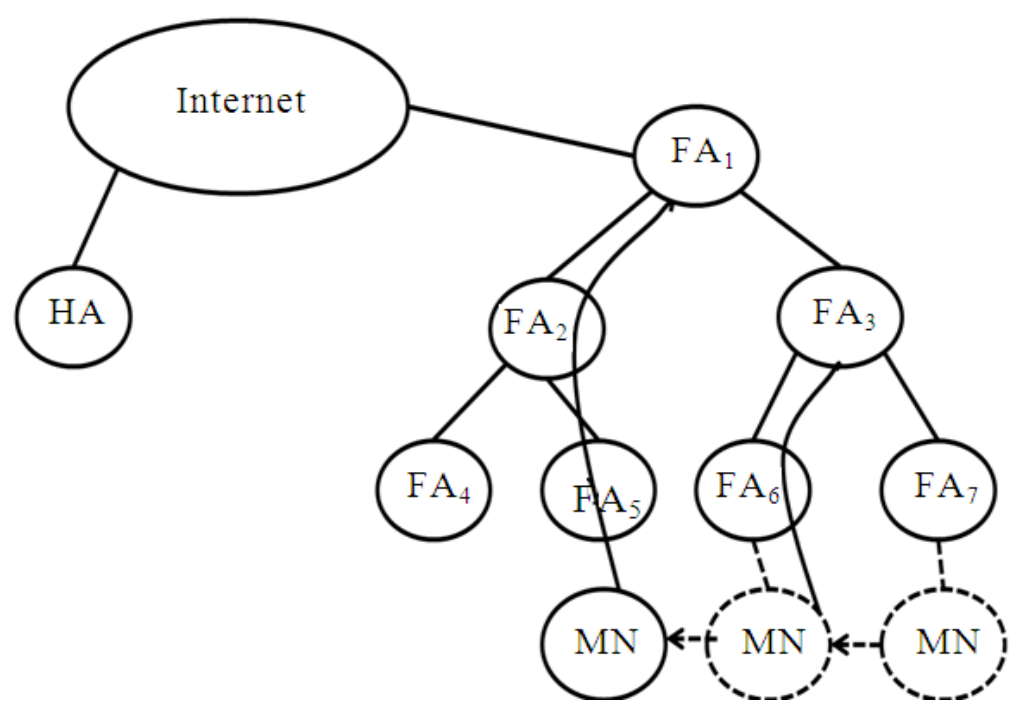

Fig. 2. Hierarchical FAs

When the $\mathrm{CN}$ sends a packet to the MN, HA intercepts it, encapsulates and tunnels it to the root of the FA hierarchy. FA re-tunnels it to the FA at its lower level and finally it gets delivered to the MN by the FA at the lowest level.

Perkins and Wang (1999) Proposes a foreign agent buffering mechanism to avoid packet loss during the handover process. The old FA buffers the packets during the handover process and when it receives previous foreign agent notification message, it re-tunnels the packet to the new FA. The efficiency of this method is based on how fast the MN finds a new FA and the buffer size. FAs can send out the beacons more frequently so that $\mathrm{MN}$ can find the new FA quickly which in turn wastes the bandwidth resources. Larger buffer can store more packets but it is an overhead in FA.

Liu et al. (2007) proposes to use a Mobility Management System (MMS) by exploiting Domain Name System (DNS) so that the functionality of mobility management is transparent. Packet flow between the MN and $\mathrm{CN}$ is taken care by the MMS. $\mathrm{CN}$ sends the data packet to the MMS which will then deliver the packet to the $\mathrm{MN}$ and vice versa. Both $\mathrm{CN}$ and $\mathrm{MN}$ are not aware of the existence of MMS. 


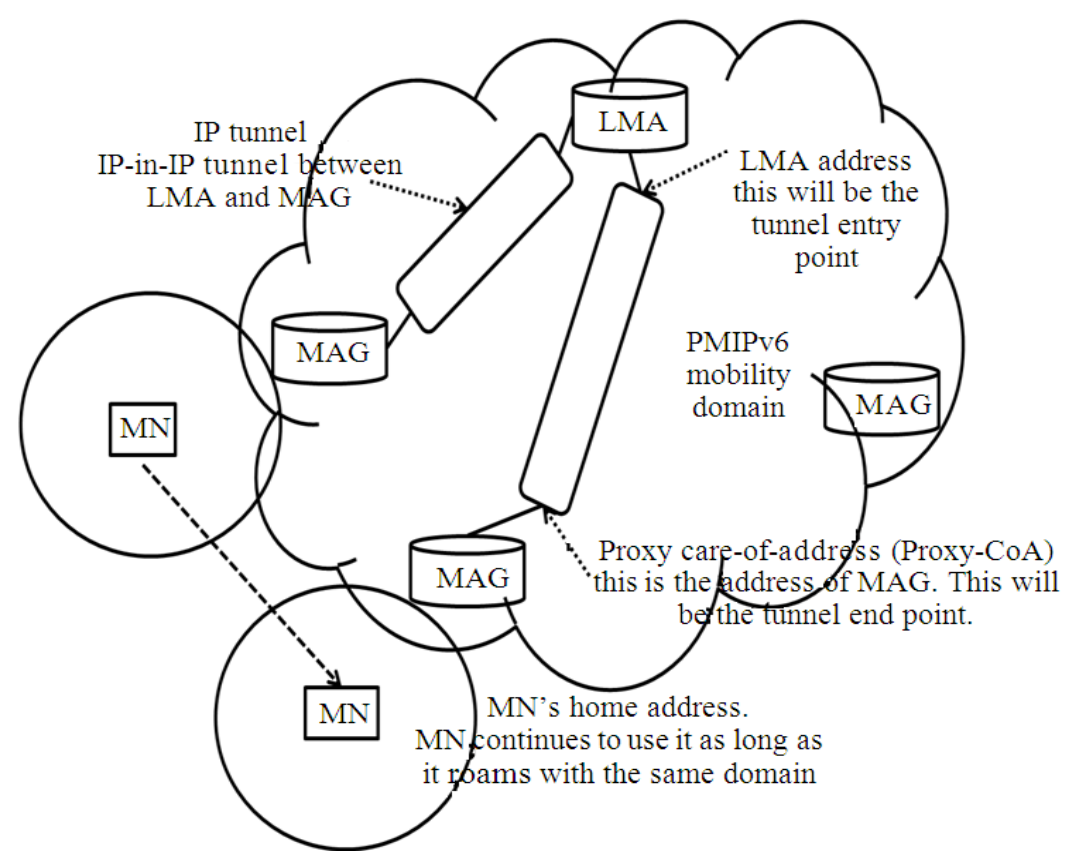

Fig. 3. Overview of PMIPv6

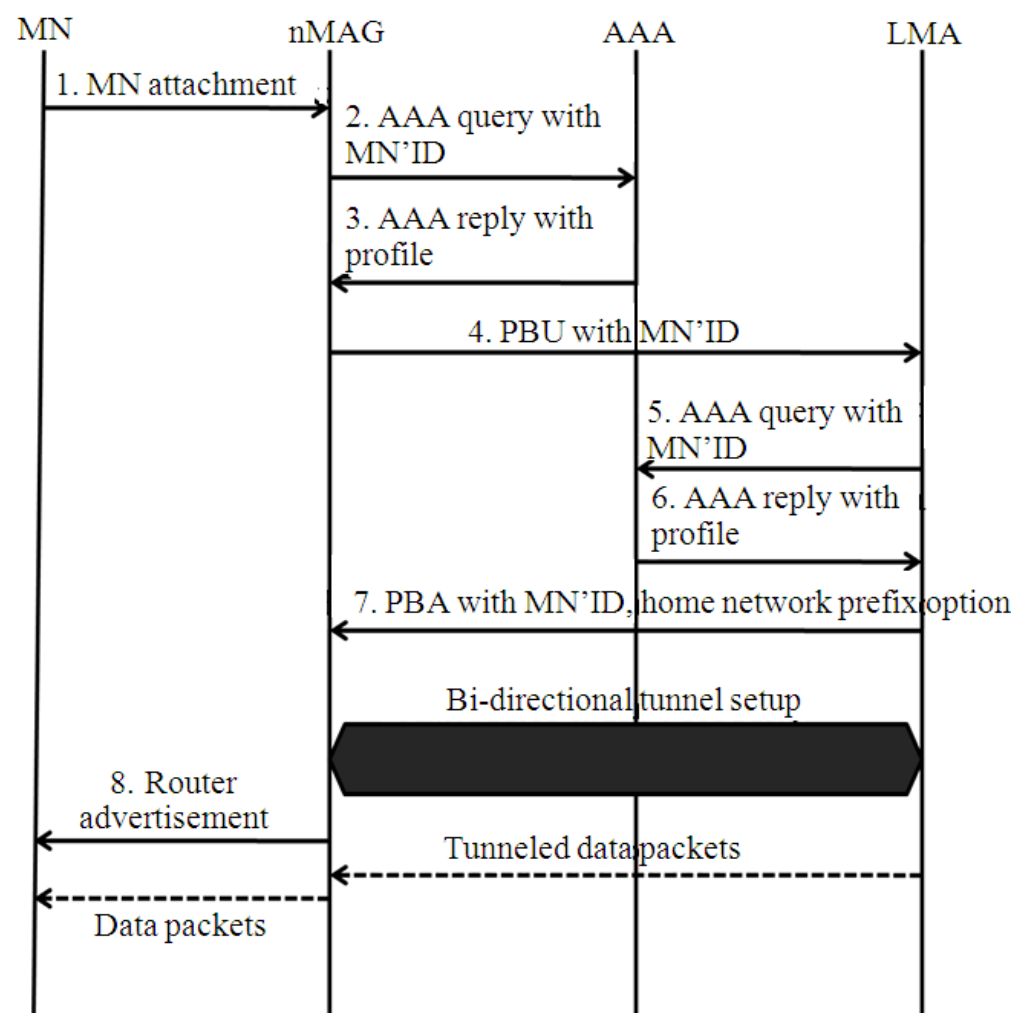

Fig. 4. Message flow in basic handover for PMIPv6 
The MMS must be chosen in such a way that it is located on the straight path between the $\mathrm{MN}$ and $\mathrm{CN}$. In other words, it must be located either near the foreign agent or in the domain zone of the CN. If the MMS located near the home agent of the MN is chosen, then it increases the path length and introduces triangle routing problems.

Lei and Fu (2008) proposes the handover process based on GPS Information. It introduces a new network entity called Mobility Controller (MC). MC contains all details of the registered Access Points (AP) in its database. MC calculates the distance between the MN and its current AP periodically and when the distance is more than the configured threshold value, it chooses an $\mathrm{AP}$ which is nearer to the $\mathrm{MN}$ and sends Handover Initiate (HI) message to the MN containing the details of the new chosen AP. All the MNs are configured with a GPS receiver which is an overhead to the MNs and the proposed method could not perform well when the speed of the $\mathrm{MN}$ is high.

Li et al. (2008) propose to maintain a new CoA table which has fields like Active, Start Time and Valid Time at each Access Router (AR). When the handover anticipation trigger occurs, Previous Access Router (PAR) sends the information about the MN to the New Access Router (NAR). NAR will generate a new CoA based on the information of the MN it has received and does the Duplicate Address Detection (DAD) process. If the DAD process fails, a new CoA is generated without using the information of the MN or if the DAD process succeeds, the generated $\mathrm{CoA}$ is inserted into the CoA table and the Active field is marked as False (F). The Active field is marked as Active (A) when the CoA is currently being used. Each AR must maintain a CoA table which is an additional overhead.

In network based management such as PMIPv6, the serving network controls the mobility management on behalf of mobile nodes; hence the tunnelling overhead and the signal-related message exchanges via the message links can be greatly reduced. PMIPv6 has many advantages; it does not need any modifications in mobile nodes, supports both IPv4 and IPv6, avoids tunnelling overhead and improves handover performance.

The rest of the study is organized as follows: Section II gives a brief introduction of PMIPv6 and its related work. Section III describes the proposed work. Section IV presents the performance analysis of the proposed method. Section $\mathrm{V}$ concludes the study and gives a brief description about our future work.

\subsection{Related Work}

PMIPv6 is a network-based mobility management protocol in which the serving network controls the mobility management on behalf of the mobile nodes. Local
Mobility Anchor (LMA) and Mobile Access Gateway (MAG) are the two main functional entities that are responsible for IP mobility for a MN in PMIPv6 domain.

MAG is mainly responsible to keep track of MN's movements and initiate the mobility-related signaling with the MN's LMA for handover process. MAG also establishes a shared tunnel with the LMA for enabling the $\mathrm{MN}$ to use an address from its home network prefix and emulates the MN's home network on the access network for each MN.

LMA is similar to the home agent in MIPv6. It also has some additional functionality in PMIPv6. The main functionality is to reach the MN when it moves within the PMIPv6 domain. From the MN perspective, the PMIPv6 domain appears as a home network. LMA also maintains a binding cache entry in which the MN and the MAG under which it is associated are mapped. This helps to maintain the relationship between the MAG and the LMA.

Figure 3 illustrates the packet delivery mechanism in PMIPv6. The packets to the MN are intercepted by the LMA in a PMIPv6 mobility domain. LMA establishes a shared IP-in-IP tunnel to the MAG under which the MN is associated. LMA address is the address at the tunnel entry point and the address of MAG is the address of the tunnel endpoint. LMA encapsulates and tunnels the packet to the MAG which decapsulates and delivers the packets to the MN.

When the MN moves from the previous MAG (mag) to the new MAG (nMAG), the basic handover process (Luo et al., 2011) is illustrated in Fig. 4. The steps are as follows:

Step 1: When MN attaches to nMAG, it is authenticcated using its identifier. nMAG knows MN's identity after successful authentication.

Step 2: The nMAG sends a query to the policy store (e.g., authentication, authorization and accounting (AAA) server) to get MN's configuration profile.

Step 3: MN's identifier, its LMA address and supported address configuration mode are sent to nMAG by the policy store.

Step 4: nMAG sends the Proxy Binding Update (PBU) message to the LMA along with the MN's identifier.

Step 5: When the LMA receives the PBU message, it checks with the policy store to ensure that the sender (nMAG) is authorized to send the PBU message.

Step 6: The policy stores replies back to the LMA with the result of authorization. 


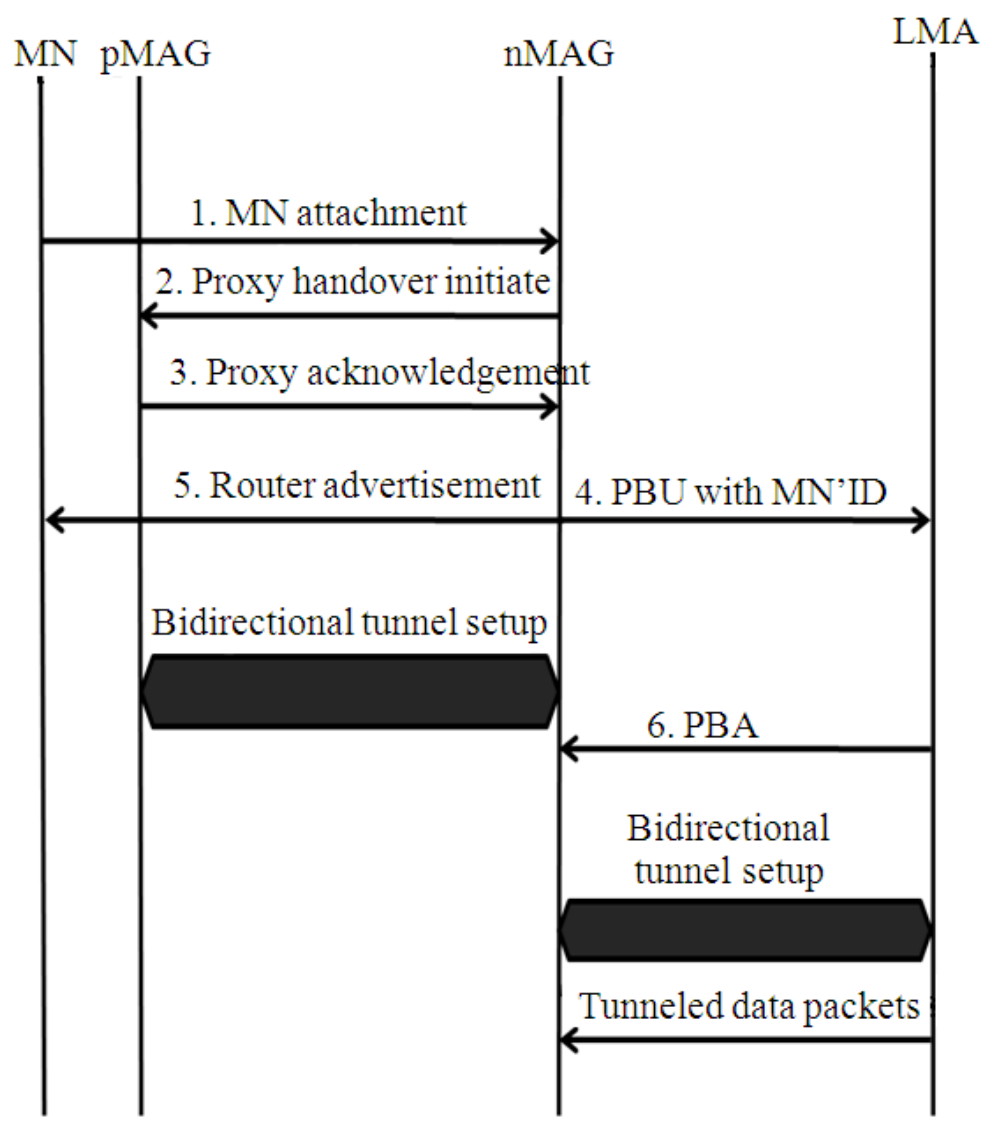

Fig. 5. Message flow in Fast Handover for PMIPv6

Step 7: If the authorization result is positive, then the LMA updates it binding cache entry and sends Proxy Binding Acknowledgement (PBA) message along with the MN's home network prefix option and establishes a tunnel to nMAG. The addresses at the endpoints of the tunnels are the LMA address and nMAG ad-dress.

Step 8: nMAG also sends a Router Advertisement (RA) message to the MN and sets up a tunnel to the LMA.

Step 9: Any subsequent packets from the Correspond in Node $(\mathrm{CN})$ is tunneled from LMA to nMAG which then delivers it to the $\mathrm{MN}$.

Lei and $\mathrm{Fu}$ (2008) proposes the fast handover mechanism of PMIPv6 illustrated in Fig. 5. The steps are as follows:

Step 1: When MN attaches to nMAG, it is authenticcated using its identifier. nMAG knows MN's identity after successful authentication. Similar to Step 1 of basic handover

Step 2: nMAG sends the Proxy Handover Initiate (PHI) along with the MN's identifier to the pMAG. In addition, it also sends the Proxy Binding Update (PBU) message along with MN's identifier to the LMA in order to update the MN's new location.

Step 3: On receiving the PHI message from nMAG, pMAG sends the Proxy Acknowledgement (PA) message which includes MN's identity, LMA's address, MN's supported address configuration mode and MN's home network prefix option.

Step 4: When nMAG receives the PA message from pMAG, it emulates MN's home network and sends the Router Advertisement (RA) mes sage to $\mathrm{MN}$.

Step 5: Data packets are tunnelled from LMA to pMAG which is then tunnelled to nMAG. nMAG delivers the packet to the $\mathrm{MN}$. 


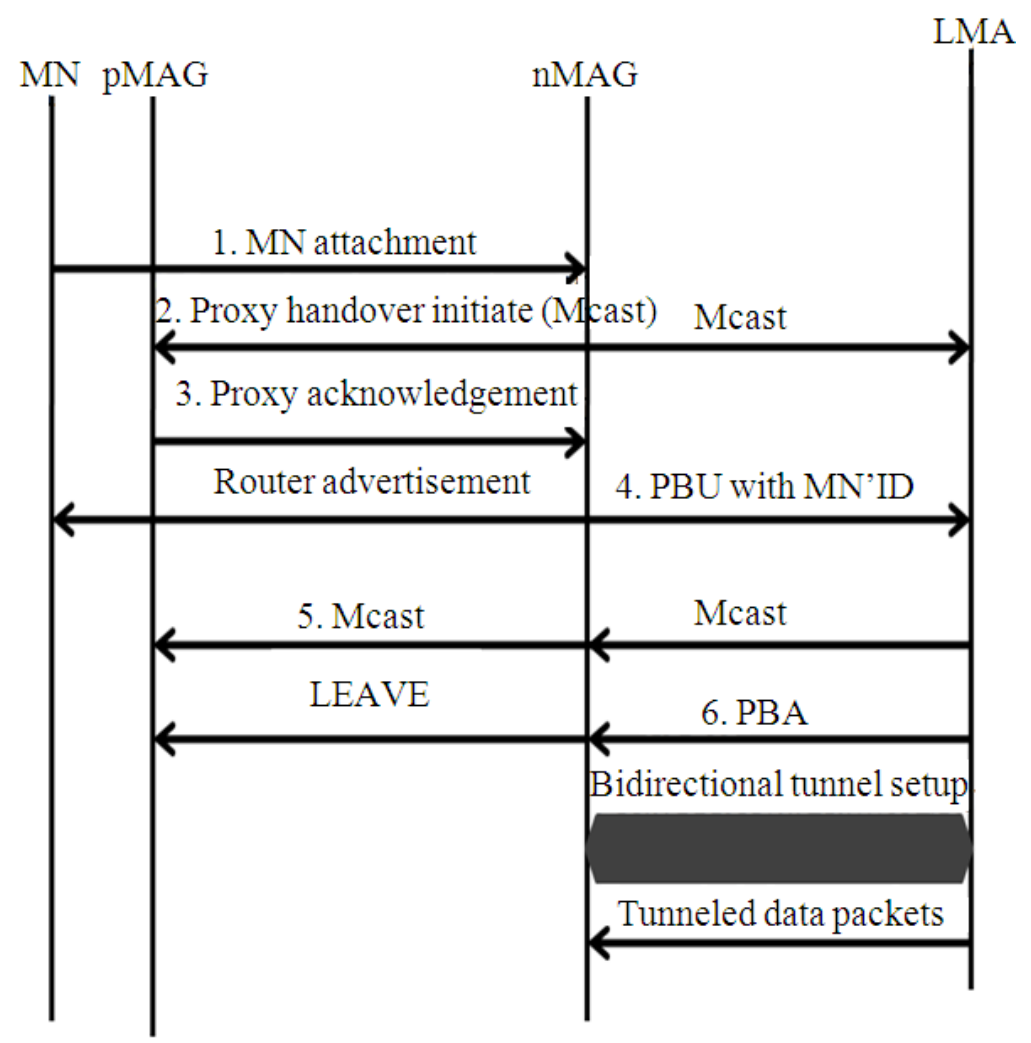

Fig. 6. Message flow in proposed fast handover for PMIPv6

Step 6: After receiving the PBU message from nMAG, LMA updates its binding cache entry and sends PBA message to nMAG. In addition, it establishes a tunnel to nMAG.

Step 7: Subsequent data packets are tunnelled directly to nMAG by LMA. nMAG delivers the packet to the MN.

This method reduces the handover delay but wastes the bandwidth resources as a bi-directional tunnel is established between the pMAG and nMAG during the handover process.

Luo et al. (2011) propose that every MAG itself also functions as a LMA. SHA-1 is used to map the MN and its associated MAG in the LMA. The MAG which serves the MN for a longer time, the one in MN user's home or work place, is chosen to function as a LMA. In such cases, the packets need not be tunnelled between the LMA and the MAG. Instead MAG itself can deliver the packets to and from the MN. But this method is not suitable for a constantly moving $\mathrm{MN}$.

\subsection{Multicast-Supported Fast Handover}

\subsubsection{Overview}

In this study, we propose a method to multicast the data packets to both the pMAG and nMAG instead of establishing the tunnel between pMAG and nMAG which wastes the bandwidth resources. nMAG triggers the LMA to multicast the data packets and then LMA switches to multicast mode. pMAG and nMAG also joins the multicast tree rooted at the LMA. After the completion of the handover process, pMAG, nMAG and LMA switches back to unicast mode. Hence the proposed method eliminates the wastage of bandwidth resources and delivers the packet efficiently during the handover process (Lai and Shieh, 2009). LMA maintains the binding cache entry of the list of MAGs and its associated MNs which are hashed using SHA-512 algorithm so that the associated MAG can be retrieved quickly using the message digest of the MN's address. SHA-512 is more secure than the SHA-1 algorithm which suffers from security flaws due to mathematical weakness.

\subsection{Algorithm}

Figure 6 illustrates the message flow of the proposed fast handover for PMIPv6. The steps are as follows: 
Step 1: When MN attaches to nMAG, it is authentic cated using its identifier. nMAG knows MN's identity after successful authentication. Similar to Step 1 of basic handover

Step 2: nMAG sends the PHI message to the pMAG with the MN's identifier and augmented multicast address. In addition, nMAG also sends the multicast address to the LMA.

Step 3: When pMAG receives the augmented PHI message, it switches to multicast mode. It replies back with PA message with MN's identity, LMA's address, MN's supported address configuration mode and MN's home network prefix option.

Step 4: On receiving the multicast address, LMA switches to multicast mode and multicasts subsequent data packets to both the pMAG and nMAG.

Step 5: After receiving the PA message, nMAG sends the RA message to the MN and also sends the PBU message along with MN's identifier to the LMA in order to update its binding cache entry.

Step 6: After updating its binding cache entry, LMA replies back to nMAG with PBA message. LMA also sets up a tunnel with the nMAG. LMA also sends LEAVE message to pMAG triggering it to switch back to unicast mode.

\section{DISCUSSION}

In F-PMIPv6 the pMAG buffers the packets during the handover process. In our proposed method, pMAG switches to multicast mode on the reception of PHI message. Packets are delivered to the $\mathrm{MN}$ through pMAG using multicast address until the completion of L2 handover. Hence there is no need to buffer the packets in pMAG and a bi-directional tunnel need not be established between pMAG and nMAG reducing the bandwidth usage.

In F-PMIPv6, after the completion of handover, nMAG receives packets from pMAG through the bidirectional tunnel between them and also from LMA. Packets from pMAG arrive later than the reception of packets from LMA. Hence it suffers from out-of-order problem (Lai and Shieh, 2009), the out-of-order packets from pMAG arises confusion in real-time applications and degrades the performance. But in our proposed method, pMAG and nMAG are attached to the multicast tree with LMA as the common ancestor node. Packet flow to nMAG and pMAG will have a significantly smaller leap time. Hence our proposed method is less vulnerable to out-of-order problem.

\subsection{Performance Analysis}

OMNET++ is an object-oriented modular discrete event network simulator. MiXiM (Mixed Simulator) is an $\mathrm{OMNeT}++$ modeling framework created for mobile and fixed wireless networks. It offers detailed models of radio wave propagation, interference estimation, radio transceiver power consumption and wireless MAC protocols. We have tested our proposed method in OMNET++ along with MiXiM framework for mobility. Constant Speed Mobility is chosen to simulate our proposed method.

The handover latency is compared with the basic handover latency for PMIPv6 by considering the factors that affect them. The notations (Montavont and Noel, 2006) for these factors are as follows:

- The average time delay for a packet to be sent between MN and the MAG is $t_{\mathrm{mm}}$

- The average time delay for a packet to be sent between two MAGs is $t_{a m}$

- The average time delay for a packet to be sent between the MAG and the AAA is $t_{a}$

For basic handover in PMIPv6, the average handover delay is given by (Montavont and Noel, 2006) Equation 1 and 2:

$\mathrm{D}_{\mathrm{standard}}=4 \mathrm{t}_{\mathrm{a}}+2 \mathrm{t}_{\mathrm{am}}+\mathrm{t}_{\mathrm{mm}}$

For proposed fast handover, the average handover delay is given by:

$\mathrm{D}_{\text {fast }}=2 \mathrm{t}_{\mathrm{am}}+\mathrm{t}_{\mathrm{mm}}$

Figure 7 shows the test bed in our simulation. The PMIPv6 domain consists of four MAGs and 21MNs are associated to any one of these MAGs according to their position. LMA consists of the details of all these four MAGs and the MNs associated with them. LMA maintains a binding cache entry in which the ipv6 address of the MN is hashed using SHA-512 algorithm and mapped against the associated MAG's address.

Figure 8 shows the comparison of handover delay of the basic handover and the proposed fast handover method by varying $t_{a m}$ when $t_{m m}=12 \mathrm{~ms}$ and $t_{a}=10 \mathrm{~ms}$. It can be clearly seen that the handover latency of the proposed method is much lower than the handover latency of the basic handover method for PMIPv6. 


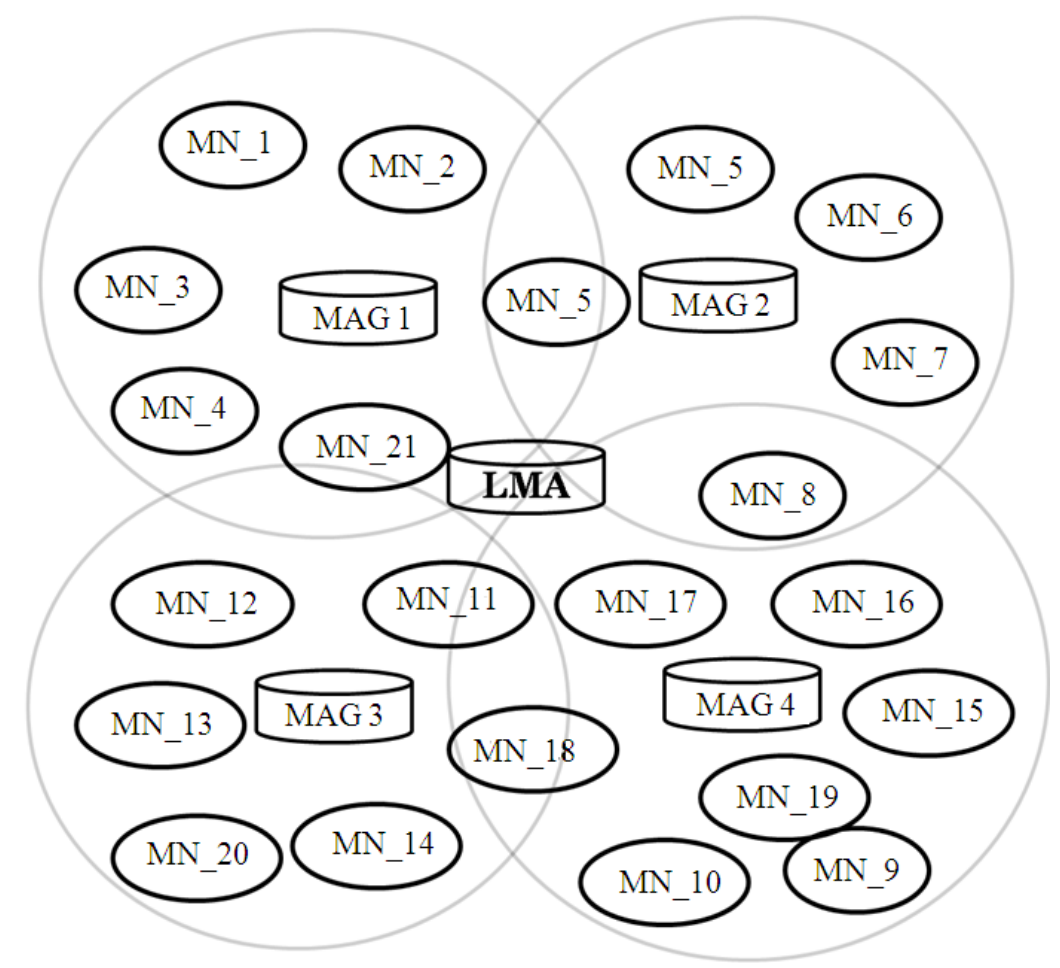

Fig. 7. Network topology test bed

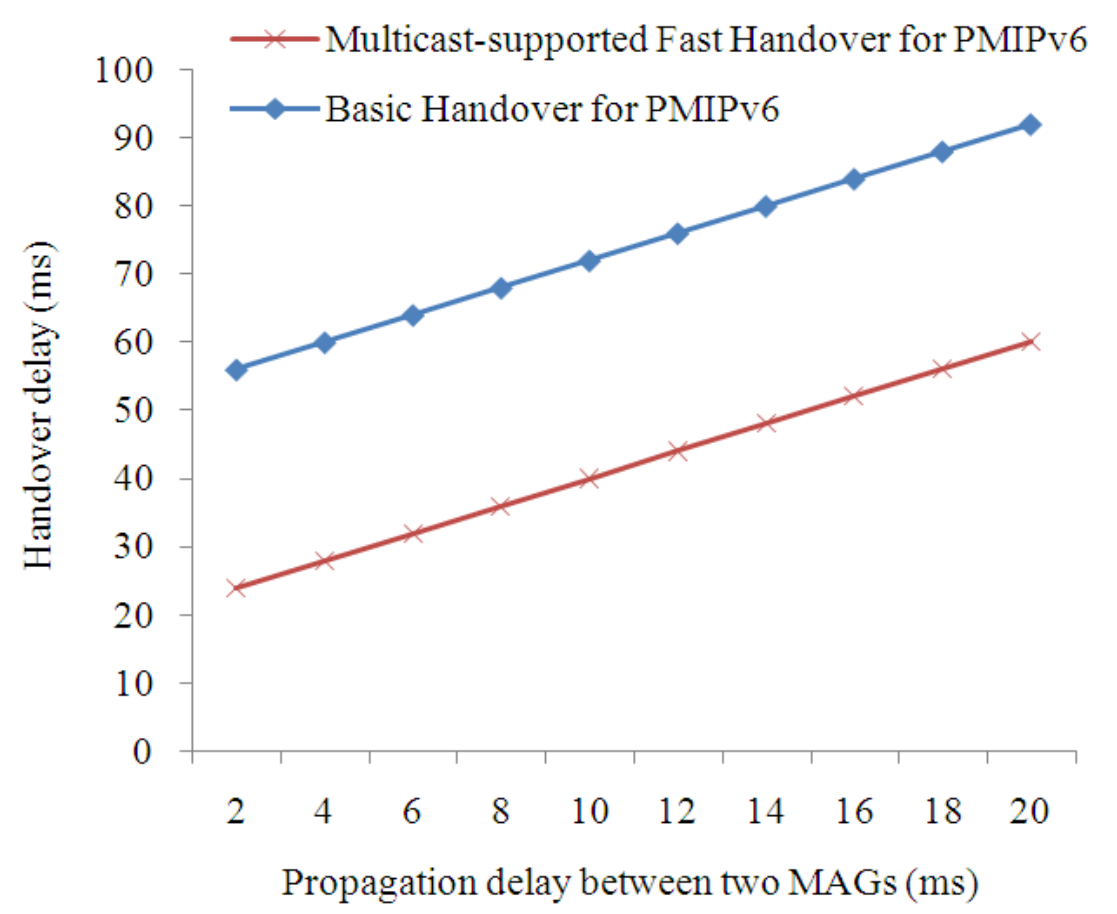

Fig. 8. Comparison of handover latency when $t_{m m}=12 m s$ and $t_{a}=10 \mathrm{~ms}$ 


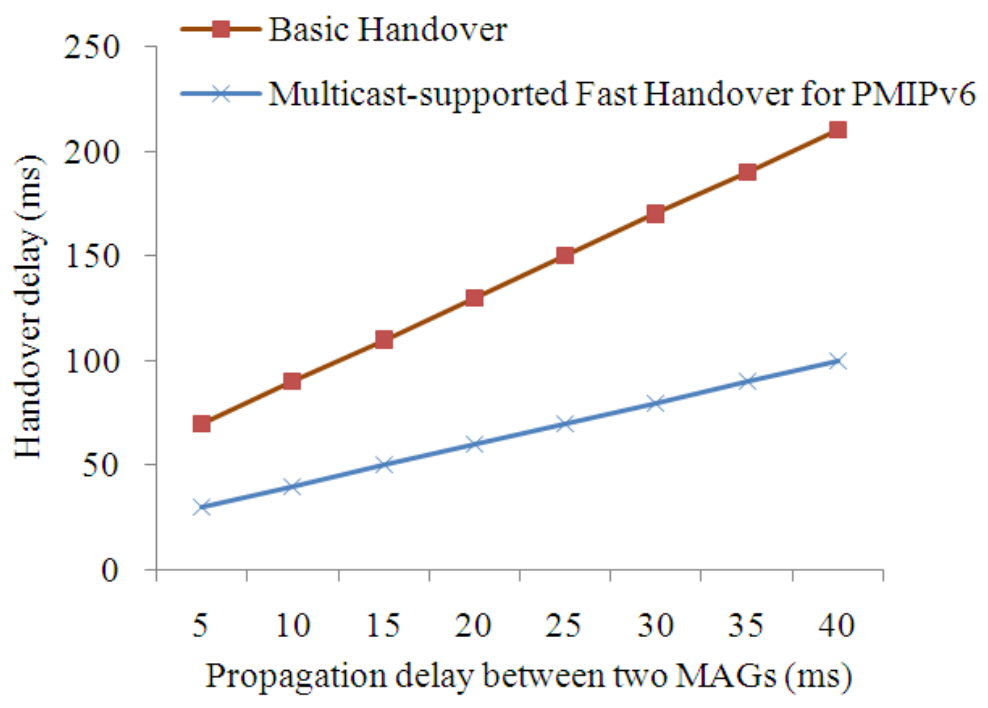

Fig. 9. Comparison of handover latency when $t_{\mathrm{mm}}=12 \mathrm{~ms}$ and $t_{\mathrm{am}}=10 \mathrm{~ms}$

Figure 9 shows the comparison of handover delay of the basic handover and the proposed fast handover method by varying $t_{a}$ when $t_{m m}=12 \mathrm{~ms}$ and $t_{a m}=10 \mathrm{~ms}$. The figure clearly shows that the handover latency of the proposed method is much lower than the handover latency of the basic handover method for PMIPv6.

\section{CONCLUSION}

This study proposes multicasting the data packets during handoff from the LMA to the nMAG and pMAG by eliminating the tunnel establishment between them. This reduces the handover delay and packet loss. The performance and the latency periods are graphed and compared with the basic handover of PMIPv6 and are proved to be better. Though the graph is not shown for fast Handover for PMIPv6 it is understood that the proposed method outperforms the fast Handover for PMIPv6.

\section{REFERENCES}

Lai, W.K. and C.S. Shieh, 2009. Improving handover performance by switching between unicast and multicast addressing. IEEE Trans. Wireless Commun., 8 : $1238-1246 . \quad$ DOI: 10.1109/TWC.2008.070149

Lei, J and $\mathrm{X} . \mathrm{Fu}, 2008$. Evaluating the benefits of introducing PMIPv6 for localized mobility management. Proceeding of the International Wireless Communications and Mobile Computing Conference, Aug. 6-8, IEEE Xplore Press, Crete Island, pp: 74-80. DOI: 10.1109/IWCMC.2008.14
Li, R., J. Li, K. Wu, Y. Xiao and J. Xie, 2008. An enhanced fast handover with low latency for mobile IPv6. IEEE Trans. Wireless Commun., 7: 334-342. DOI: 10.1109/TWC.2008.060582

Liu, H.J., C.H. Wang and C.L. Tseng, 2007. Transparent IP Mobility Management. Future Generation Commun. Netw., 1: 374-377. DOI: 10.1109/FGCN.2007.222

Luo, H., H. Zhang, Y. Qin and V.C.M. Leung, 2011. An approach for building scalable proxy mobile IPv6 domains. IEEE Trans. Network Service Manage., 8: 176-189.

DOI: 10.1109/TNSM.2011.071511.20100063

Montavont, $\mathrm{M}$ and $\mathrm{T}$. Noel, 2006. IEEE 802.11 handovers assisted by GPS information. Proceedings of the 2nd IEEE International Conference on Wireless and Mobile Computing, Networking and Communications, Jun. 19-21, IEEE Xplore Press, Montreal, Que., pp: 166-172. DOI: 10.1109/WIMOB.2006.1696358

Perkins, C.E. and K.Y. Wang, 1999. Optimized smooth handoffs in mobile IP. Proceedings of the International Symposium on Computers and Communications, Jul. 6-8, IEEE Xplore Press, Red Sea, pp: 340-346. DOI: 10.1109/ISCC.1999.780874 